NOTICE: this is the author's version of a work that was accepted for publication in Assessment and Evaluation in Higher Education. Changes resulting from the publishing process, such as peer review, editing, corrections, structural formatting, and other quality control mechanisms may not be reflected in this document. Changes may have been made to this work since it was submitted for publication. The definitive version can be found at http://dx.doi.org/10.1080/02602938.2010.488797

To cite this article: Anna Sutton \& David Taylor (2011) Confusion about collusion: working together and academic integrity, Assessment \& Evaluation in Higher Education, 36: 7, 831-841

\title{
Confusion about collusion: working together and academic integrity
}

Anna Sutton ${ }^{\mathrm{a}}$ and David Taylor ${ }^{\mathrm{b}}$

${ }^{a}$ Manchester Metropolitan University Business School, Manchester, UK

${ }^{b}$ Leeds University Business School, Leeds, UK

* Corresponding Author: Anna Sutton. Email: a.sutton@mmu.ac.uk

Acknowledgements: This research was supported by grants from BMAF (Business

Management Accounting and Finance Network of the Higher Education Academy) and Leeds Metropolitan University 


\section{Abstract}

An increasing emphasis on developing students' transferable skills, such as group working and IT, is creating challenges in ensuring the academic integrity of individually assessed coursework. This study investigated the frequency with which students engaged in a range of study behaviours for individual assignments, with a focus on the extent to which they exchanged information or worked in informal study groups. Over 1,000 responses were gathered from students at pre- and post-92 universities engaged in either Business or Psychology degrees. Four behavioural factors emerged from the data: Trust, Cooperation, Use of IT and Conscientious practices. Results indicated that students engaged in practices relying on trust and cooperation less often than other practices, implying a concern with avoiding issues of academic misconduct. This was supported by focus group discussions, where students described their strategies for working together while ensuring the quality and integrity of their own work. Comparisons between academic disciplines revealed Business students were more likely to engage in sharing and group work behaviours than Psychology students, as were students at the post-92 university. Comparisons between years found no significant differences. Recommendations are made for improving student understanding of collusion while still encouraging the development of skills important to employability. 


\section{Introduction}

The traditional university approach to learning envisages the student learner as independent, predominantly using printed sources to gather and integrate information. The increased importance of provision and acquisition of transferable and employability-related skills has led to a shift of emphasis, with university learning developing students' facility in using IT and group working. Whilst the ability to demonstrate these skills is valuable in a competitive employment environment, this can create problems for course assessments. Increased group work leads to issues around collusion when students are required to complete individual assessed coursework (ACW), while increased use of IT can lead to problems with students cutting and pasting information from an original source which then appears in their final assignments. These practices create a challenge for markers: to improve students' transferrable skills with relevant assessments and learning approaches whilst maintaining assessment integrity.

\section{Literature Review}

Academic misconduct is a very widespread problem, with many authors claiming it is on the rise (reviewed in Park 2003). There is evidence that students in different academic disciplines or institutions have different levels of "cheating" behaviours, of which plagiarism and collusion are a part, and it has been suggested that the most common types of cheating behaviours tend to be those around sharing work with peers, including actions such as copying assignments or giving another student the answers (Lin and Wen 2007).

There is conflicting evidence over which students cheat more: Park's review indicates that Business students are the most likely to cheat, while Iyer and Eastman (2006) found that non-business students were more likely to cheat. Barrett and Cox (2005) suggest that psychology or law students may plagiarise less than students doing science or engineering degrees because there are less "clear cut" answers in the former disciplines, 
making direct copying less easy. Finally, Premeaux (2005) found that the majority of students at AACSB (Association to Advance Collegiate Schools of Business) accredited business schools had "cheated" in academic work at some point.

Previous work has shown that while students generally have a clear understanding about the more overt acts of academic misconduct such as buying assignments and coercing others into doing assignments, they are less clear about the distinction between legitimate collaboration and collusion (Barrett and Cox 2005, Taylor et al. 2007). Collusion may be thought of as more acceptable because there is a sense that individuals are still engaging with the material in a learning process (Barrett and Cox 2005). Supporting this view, Hayes and Introna (2005) found that students may believe that "good" plagiarism implies a certain level of understanding of and engagement with the topic, so some learning has taken place. While students can usually give a good definition of plagiarism, they are often less clear on the specific actions that could be seen as academic misconduct, particularly those that involve working with others (Yeo 2007).

The cause of this confusion can be further illustrated through Culwin and Naylor's (1995, cited in Culwin and Lancaster n.d.) description of a continuum from collaboration through collusion to copying. Collaboration is defined as working together to share information or material that may be included in the final version of an assignment. The point at which this becomes collusion (an unacceptable level of shared work in the final assignment) is difficult to define and is dependent on the norms of individual establishments or markers (Culwin and Lancaster n.d.). It is not unusual for institutions to have a very specific and rigorous definition of plagiarism but to be less clear about what constitutes collusion and delineates the boundary between what is acceptable (collaboration) and not acceptable (collusion). The Little Book of Plagiarism (Stinson 2003), produced by Leeds Metropolitan University and adapted for use at several other 
institutions to give students advice on avoiding plagiarism, is an example of this. The booklet give a clear definition of plagiarism but acknowledges that there "are grey areas e.g. when is discussion with fellow students good practice and when does it become collusion?" There is also some discussion around how students from different countries perceive collaboration, with indications that those from more collectivist cultures may find it difficult to understand the distinction drawn between collaboration and cheating in more individualistic cultures (Robinson and Kuin 1999).

There is clear evidence that students are concerned about academic misconduct and the negative consequences that can result from it (Ashworth et al. 1997) and the concern that they may somehow "unwittingly" cheat is not unfounded. Cryptomnesia (hidden memory) is psychological phenomenon whereby people can believe they have produced an original idea when in reality they are recalling something that was presented to them before (Park 2003). Recent work indicates that the credibility of the source and the act of generating improvements to ideas both have independent effects on unconscious plagiarism. A more credible source of the original ideas leads to more instances of unconscious plagiarism when participants are asked to come up with new ideas; while tasks involving improving on another's ideas lead to more instances of participants believing the ideas were their own rather than their partner's (Perfect et al. 2009). This has implications for students who collaborate on work for which they are given independent grades and demonstrates that students who are concerned that they may unwittingly appropriate someone else's ideas have good grounds for this belief.

How staff should deal with academic misconduct is a thorny issue as not only is there a variation in how individual staff view this (Flint et al. 2006), but there is also evidence that a very strict approach will actually discourage honest students who fear unconscious plagiarism as well as those who deliberately plagiarise (Levy and Rakovski 
2006). Many institutions have severe penalties for acts of academic dishonesty, including exclusion from the course. Yet Ashworth et al (1997) found that students often place values about friendship and trust above the institutional rules about plagiarism, so that they may justify collusion to themselves on the basis of "helping out their friends". Students may also copy work because of their strong desire for a good grade coupled with the belief that they are incapable of attaining this legitimately, perhaps due to time pressures or lack of knowledge (Robinson and Kuin 1999). However, Ashworth's study also found that students have a strong ethical view on the importance of learning and being fair to their peers which the authors suggested could be built upon to develop student understanding of academic misconduct, and, more specifically, what is expected of them in the area of working together.

Working with others is an essential skill in the modern workplace and the majority of graduate level jobs expect applicants to be able to demonstrate their team working ability. Analysis of graduate employer surveys shows that "People skills"(including team working, interpersonal skills and communication) is one of the four major categories of skills the employers expect new graduates to offer (Graduate Prospects Ltd 2008). Giving students experience of working with others to achieve a common outcome is an essential means of developing these valuable skills.

Collaboration is also increasingly important as a part of knowledge sharing and building communities in academia and business and something that students engage in informally whether or not their courses formally expect them to (Hodgkinson 2006). It is important that lecturers understand how students work together on an informal level if they are to build courses and assessments that work with the students' strengths and take account of any potential difficulties students may come across. As an example, students 
working in informal study groups for independent ACW may make unwitting (or deliberate) collusion more likely.

Universities and lecturers are in a difficult position. On the one hand, they want to improve students' transferrable skills and their chances of employment and on the other, they need to ensure the integrity of the assessment procedures. This study provides information which will assist educators to resolve this impasse. It explores and categorises how students today approach individual ACW, how they work together, and to what extent they share information in doing so, as well as the strategies they use to ensure the integrity of their academic work. By quantitative evaluation of the behaviours students engage in and further exploring the results through focus groups, this study categorises the strategies already in use into wider themes and makes recommendations for educators. This will assist educators in facilitating students' development of strategies for informal group working and peer interactions and recognise which of these are appropriate for individual ACW.

\section{Method}

A questionnaire was developed through initial focus groups and feedback from experts in the area. The questionnaire assessed how often students did 13 actions related to their university work, including taking part in informal study groups, using IT in preparing notes and final assignments, and sharing their work with others. Responses were on a five point frequency scale from 1 (never), 2 (occasionally) 3 (often) 4 (mostly) to 5 (always).

The questionnaire was administered to undergraduate students from three different schools at two universities in the same northern English city: 208 Business and 207 Psychology students from Institution A (a post-92 university) and 623 Business students from Institution B (a pre-92 university in the same city). The distinction is drawn between institutions that gained the title of university before or after The Further and Higher 
Education Act 1992 as these are, broadly speaking, institutions with a different ethos. Post92 universities typically place greater emphasis on the vocational nature of the programmes they offer with commensurately greater focus on direct teaching activity; pre92 universities typically identify themselves as "research-led" or "research intensive" with greater focus on research activity and hence a different teaching approach. This differentiation is widely acknowledged and known to candidates prior to application, hence a central theme of the project was comparison of the data for the two institutions on the basis that the study skills profile of students may differ and explore whether this position would be reflected in the findings.

The questionnaire was distributed and collected in module classes; a methodology widely used in the institutions concerned, for example for module review questionnaires. This methodology was selected as students are familiar with it and it achieves a high response rate. Participation was entirely voluntary, the questionnaire was anonymous and no personal information was elicited. In all 1038 responses were received.

After results were analysed, focus groups were conducted with students from the Institution A Psychology $(\mathrm{N}=10)$ and from Institution B Business $(\mathrm{N}=7)$ to explore whether the statistical results aligned with students' views. Participation was voluntary and students were given a voucher in exchange for their time. Students who attended these groups were likely to be inherently interested in the topic and may not represent the views of the whole cohort.

\section{Results}

\section{Questionnaire analysis (quantitative data)}

The most significant contribution of this paper to the literature is that rather than compare responses on individual items, a factor analysis was conducted. This approach, which as far as the authors are aware has not been used in this area before, enables a 
thematic structure to emerge from the statistical treatment of the responses to individual questions as opposed to being artificially constructing by grouping questions together under broader sub-headings. Use of factor analysis legitimises the emergent thematic structure of grouping of the responses to individual questions (examination of the "themes" indicates the questions grouped together intrinsically have common ground).

The items were subjected to principal components analysis with varimax rotation. Four factors emerged with eigenvalues greater than 1 . Table 1 shows the rotated factor loadings for each item.

----Table 1 about here----

The first factor (Trust) clearly groups those items which are to do with interactions with individuals based on a sense of trust with the other, such as sharing information with others and believing the shared information will not be misused or the trust betrayed. It is interesting to note that the item about exchanging word processed assignments loads nearly as strongly on the second factor, which is about working with others.

The second factor (Cooperation) groups items to do with working with others to gather or share information. This includes informal study groups and virtual groups (e.g.: Facebook).

The third factor (Use of IT) includes items related to how students prepare their assignments, specifically the extent to which they use word-processing tools and electronic sources.

The final factor (Conscientious working) includes students who make notes and ask for help with proof-reading. Note that the item about proof-reading also loads strongly on the Trust factor. 
Item scores were combined to produce mean scores for the three factors (negatively loading factors were reversed scored before the mean was produced). Table 2 shows the descriptive statistics for these factors.

----Table 2 about here----

On the whole, students rarely engaged in actions which could lead to collusion ("Trust" and "Cooperation" means of 1.51 and 1.57 respectively). This could reflect the continuing individual nature of university work, students' concern over collusion and a generally competitive approach to study. They occasionally (mean 2.19) completed assignments entirely on the computer, with no hand-written drafts or notes. This reflection of the increasing reliance on IT in student learning may cause concern where students do not adequately acknowledge their sources. The most common practices (mean 3.12) were those designed to help students submit good quality work, such as making notes from sources and asking for proof-reading help.

\section{Comparisons}

A MANOVA was conducted to determine whether students in different Schools and Years had different approaches to their work. The four factors (Group Work, Trust, Use of IT, Conscientious) were used as dependent variables and two independent variables (School with three levels: Psychology A, Business A and Business B; and Year with three levels:1, 2 or 3). Assumption testing using Mahalanobis distance revealed 15 multivariate outliers, which were excluded from further analysis. Box's $M$ was significant $(F=3.06, p$ $=0.001$ ), although as it is considered too strict for large sample sizes (Tabachnick and Fidell 2001) no alterations were made to the data. Levene's test was significant for three of the four variables $\left(\right.$ Trust $\mathrm{F}_{8,1010}=10.91, \mathrm{p}=0.001$; Group Work $\mathrm{F}_{8,1010}=4.32, \mathrm{p}=0.001$; Use of IT $F_{8,1010}=4.45, p=0.001$; Conscientious $\left.F_{8,1010}=1.92, p=0.054\right)$. Tabachnick and Fidell (2001) recommend using a more conservative alpha level in this case, so 
$\mathrm{p}<0.025$ was used for univariate analyses for the first three factors. They also recommend using Pillai's trace for multivariate tests if there are some violations of assumptions as it is more robust.

Analysis revealed a significant main effect of $\operatorname{School}\left(\mathrm{F}_{8,2016}=14.94, \mathrm{p}=0.001\right.$, Pillai's trace $=0.11$, partial eta squared $=0.056)$ and $Y$ ear $\left(F_{8,2016}=2.39, p=0.015\right.$, Pillai's trace $=0.02$, partial eta squared $=0.009$ ) on the reported frequency of behaviours. The interaction of School and Year was not significant $\left(\mathrm{F}_{16,4040}=1.31, \mathrm{p}=0.183\right.$, Pillai's trace $=0.021$, partial eta squared $=0.005$ ). All of these were small effect sizes according to Cohen's (1988) guidelines.

After Bonferroni corrections were applied to the stricter significance level referred to above, differences were found between Schools on Trust $F_{2,1010}=40.18, p=.001$; Group Work $\mathrm{F}_{2,1010}=12.91, \mathrm{p}=.001$ and Use of IT $\mathrm{F}_{2,1010}=8.68, \mathrm{p}=.001$; but not Conscientious $\mathrm{F}_{2,1010}=0.16, \mathrm{p}=.76$. Trust had a medium effect size (partial eta squared $\left.=.074\right)$, while Group Work and Use of IT had small effects (partial eta square of .025 and .047 respectively). Post-hoc comparisons using Bonferroni corrections indicated several significant differences between individual Schools on the first three factors $(p<0.001$; Mean scores are shown in Table 2). All three Schools were significantly different from each other on the Trust factor, with Institution A Psychology students engaging in these practices the least and Institution A Business students engaging in them most. Institution A Business students engaged in Group Work and Use IT practices significantly more often than students at both of the other two schools.

However, after Bonferroni correction, there were no significant differences between years on the individual factors: Trust $\mathrm{F}_{2,1010}=.55, \mathrm{p}=.579$; Group Work $\mathrm{F}_{2,1010}=$ $2.29, \mathrm{p}=.038 ;$ Use of IT $\mathrm{F}_{2,1010}=3.34, \mathrm{p}=.036$; Conscientious $\mathrm{F}_{2,1010}=1.88, \mathrm{p}=.154$ 


\section{Focus Groups (qualitative data)}

\section{General context}

In all focus groups, students reported a strong social pressure to "help" friends or colleagues with academic work whilst, conversely, experiencing a culture of competitiveness where they felt the need to protect their own interests to maximise the relative quality of their own work. Students were concerned about academic misconduct and had been told how serious it is, but felt they were not sufficiently well informed about expectations in this area to avoid possible problems.

Strategies reportedly used by students

A number of relevant strategies emerged as determining how much students shared information and who with:

1. Avoid the use of email to share ideas, use verbal discussions instead.

2. Help each other with problems, but do not allow direct copying.

3. Share only specific parts of an assignment. For example, share work which involves set details (e.g.: method section for a lab report) but not essays. Other students would share references but not notes or alternatively share the ideas but not the references. In all cases, students are ensuring that they do not simply hand over a finished piece of work.

4. Only share / work with those they trust - this was emphasised several times. They reported feeling under social pressure to provide help to those who ask but are only happy to share with those they can trust not to abuse that trust.

5. Only share work with people who actually do the work - means there is less chance of that person "just taking your work without doing their own".

6. If students did not wish to share work with others, they either referred to the strong rules on plagiarism to back up their decision, or were evasive. 
Effective learning of acceptable study practices

Students expressed the view that more "contact time" in small group tutorials would be effective in this context and would serve several functions:

- Improve general study skills

- Discussions on essay topics would put students on the right track when answering, as well as develop their skills at analysis and idea development

- Social support from other students, especially in the first year. This could actually reduce pressure on staff as the students will be able to help each other.

One way in which contact time could be utilised is through facilitated discussion about what is or is not acceptable by which students could explore their own position and develop individual strategies appropriate for their learning style. In the course of discussions these individual approaches could be tested to ensure they are acceptable.

A strong theme was that students wanted guidance on what was right rather than being repeatedly told what was wrong. Overall, they are looking for clear guidance on best practice rather than warnings not to do things and examples of what not to do. This is a particular challenge as this clear guidance as to what is acceptable in cooperative endeavour with others in individual assessed coursework does not appear to exist.

\section{Discussion}

Given the high level of concern about academic misconduct both anecdotally and in the literature (e.g.: Park 2003), students' self-reports about behaviours that may lead to collusion were surprisingly low. Overall, students never or only occasionally engaged in informal group work or sharing their work, even with others they trusted, possibly a reflection of the individual nature of university work and students' concern over collusion. However, even exchanging work occasionally creates a situation which could lead to collusion or copying, particularly where the format of the work lends itself to direct 
copying (for example as an e-mail attachment or by exchanging a transferable data storage device such as a flash drive or memory stick). Anecdotal evidence from recent cases of academic conduct involving collusion suggests this is becoming an issue.

Of equal concern is the indication that students occasionally completed assignments entirely online and cut and paste text into notes and, particularly, final assignments. Reported behaviours in this category still showed a low incidence, but increased and more widespread use of plagiarism detection software shows, in the experience of the authors, that the practice of cutting and pasting text and working online is more widespread than might be thought and potentially a source of difficulties. This can lead to issues about attribution or copying from sources even where the assignment is wholly the effort of one student.

In cases of suspected collusion this can make demonstrating individual contributions more difficult in the absence of previous drafts; particularly so where students work together on an individual assignment online and cutting and pasting text as a means of note-taking and building the assignment. Working in this way is not inherently collusion, but requires a very clear understanding of what is acceptable and what is not if an accusation of collusion is to be avoided.

It is promising to note that the most frequent behaviours were those designed to help students submit good quality work, such as making notes from sources and asking for proof-reading help. This pattern of results clearly shows students' genuine concern with producing independent, quality work and may well be a reflection of Ashworth et al's (1997) finding that students value learning and the importance of fairness in their assessments.

There has been much discussion in the literature about different levels of dishonesty among students in different disciplines (Park 2003) or at institutions of differing 
status (Premeaux 2005). This study explored this further by collecting data from comparable institutions on practices that could lead to collusion. Students' schools were found to have a significant effect on the frequency of various behaviours.

Firstly, psychology students engaged in sharing behaviours requiring Trust less often than business students. They also used IT less in preparing their assignments. While none of these practices is dishonest, they are practices that could lead to collusion and academic misconduct unwitting or otherwise, and it seems that psychology students may be more concerned with avoiding these practices than business students. This could imply that business students may need to be made more aware of the potential pitfalls of these behaviours. Striking the balance between warning students of the potential problems but still encouraging them to share ideas, cooperate and use IT appropriately is difficult but necessary.

Secondly, business students at a post- and a pre-92 university (Institutions A and B) in the same city were compared. Students at the pre-92 university engaged in group work and sharing behaviours less often than those at the post-92 university, possibly reflecting a more traditional approach to university working at the older institution.

Analysis of the differences between years showed no significant effects, which indicates that students are not changing their behaviours significantly from when they start university to when they graduate. This raises the question of how effective current university efforts at improving student skills in group work and collaboration really are.

\section{Conclusion and Recommendations}

Previous work has shown that students are worried about unwitting plagiarism and collusion (Barrett and Cox 2005). The situation is exacerbated by the general absence of clear guidelines as to where the boundary lies between cooperation (commendable) and collusion (unacceptable); discussions between the authors and academic colleagues 
indicate that, in line with Culwin and Lancaster's (n.d.) findings, individuals have a wide range of views as to what is acceptable in this context.

This study identified four main factors underlying student practices when completing individual assessed coursework. The lower frequencies for behaviours that are more likely to lead to collusion or plagiarism imply that students are concerned about these issues, a view supported by the qualitative data from focus groups. It is of particular note that despite voicing concern about lack of understanding of collusion, the students freely admitted that they did not use the online or printed resources available to them to learn what was appropriate, in line with previous findings demonstrating that only a third of students access online resources about plagiarism (Brown et al. 2008). Instead, students preferred a "learning by doing" approach, willing to risk getting it wrong and the penalties that brought with it rather than using the resources provided by institutions. This is a serious challenge to university educators, as the penalties can often be very severe, even to the point of the student being withdrawn from a course, and this strategy is clearly not one that the university would wish to encourage. Students emphasised the need to learn about correct referencing, collusion and plagiarism through actually completing tasks and getting feedback, rather than by being handed a booklet about it. This could be best achieved through the use of small group tutorials, where students could draw on peer support as well as the tutor's expert knowledge and give them a safe environment in which to develop their understanding of academic misconduct.

The students were aware of the dangers of collusion and were able to list several strategies they used to avoid it. The suggestions are of real use to university policy makers and lecturers as they can be used as the basis for guidance for students on how to avoid collusion but still make use of development opportunities inherent in group work. Using student suggestions as the basis for guidance, where appropriate, gives guidance more 
credence and credibility. The guidance, if publicised, can give students a "neutral" basis to help ameliorate social pressures to share work where they do not wish to do so. As noted in the qualitative data, students indicated they would share work with those they trust but recognised social pressures to exchange work more widely, with implications for friendship and acceptance in one's peer group if work was not shared. Clear guidance would provide a strong underpinning support for a refusal to share ("I am not allowed to" is a more acceptable rationale in this context than "I don't want to"). A clear policy on academic misconduct and collusion will not just protect academic integrity but also give conscientious students support when they are asked to share work where they are not comfortable with doing so.

The people skills and team working that employers are looking for cannot be developed solely in independent university work. However, balancing the need for these skills against the need for academic integrity is a fine line. This study has shown that students are aware of this difficulty, reporting a general avoidance of practices that may lead to problems in group working. However being found guilty of academic misconduct is a "low probability, high impact event" with potentially severe consequences and the generally expressed lack of clarity regarding what is acceptable practice in working together on individual assessed coursework assignments indicates that institutions would be well-advised to be proactive in developing guidance in this area. Such guidance must be clear, universally accepted by students and academic staff, and (most importantly) communicated to students in a manner whereby they both understand and appreciate the possible consequences which can be severe.

This study has developed a clear taxonomy of themes through which to address the issues arising from the challenges of working together. Educators may wish to use this taxonomy to frame targeted guidance to help students understand and avoid collusion 
while still benefiting from working together. It has also identified the strategies students use when working together on individual ACW; these can also usefully be utilised in this context. Further work is planned to explore the motivations behind exchanging work and any self-imposed limitations that students feel bound by. 


\section{References}

Ashworth, P., Bannister, P. \& Thorne, P. (1997) Guilty in whose eyes? University students' perceptions of cheating and plagiarism in academic work and assessment. Studies in Higher Education, 22, 187-203.

Barrett, R. \& Cox, A. L. (2005) 'At least they're learning something': the hazy line between collaboration and collusion. Assessment and Evaluation in Higher Education, 30, 107-122.

Brown, C., Dickson, R., Humphreys, A., Mcquillan, V. \& Smears, E. (2008) Promoting academic writing/referencing: Outcome of an undergraduate e-learning pilot project. . British Journal of Educational Technology, 39, 140-156.

Cohen, J. (1988) Statistical power analysis for the behavioral sciences, Hillsdale, NJ, Lawrence Earlbaum Associates.

Culwin, F. \& Lancaster, T. (n.d.) Plagiarism, prevention, deterrence and detection. York, Higher Education Academy.

Flint, A., Clegg, S. \& Macdonald, R. (2006) Exploring staff perceptions of student plagiarism. Journal of Further and Higher Education, 30, 145-156.

Graduate Prospects Ltd (2008) What do employers want? http://www.prospects.ac.uk/cms/ShowPage/Home_page/What_do_graduates_do 2008/What_do_employers_want/p!ebfpppd, accessed 26 June 20092009

Hayes, N. \& Introna, L. D. (2005) Cultural Values, Plagiarism, and Fairness: When Plagiarism Gets in the Way of Learning. Ethics and Behavior, 15, 213-231.

Hodgkinson, D. M. (2006) Collaborative Behaviour amongst LIS students. Education for Information, 24, 125-138.

Iyer, R. \& Eastman, J. K. (2006) Academic Dishonesty: Are Business Students Different From Other College Students? Journal of Education for Business, 82, 101-110. 
Levy, E. S. \& Rakovski, C. C. (2006) Academic Dishonesty: A Zero Tolerance professor and Student Registration Choices. Research in Higher Education, 47, 735-754.

Lin, C.-H. S. \& Wen, L.-Y. M. (2007) Academic dishonesty in higher education - a nationwide study in Taiwan. Higher Education, 54, 85-97.

Park, C. (2003) In Other (People's) Words: plagiarism by university students - literature and lessons. Assessment and Evaluation in Higher Education, 28, 471-488.

Perfect, T. J., Field, I. \& Jones, R. (2009) Source Credibility and Idea Improvement Have Independent Effects on Unconscious Plagiarism Errors in Recall and Generate-New Tasks. Journal of Experimental Psychology, 35, 267-274.

Premeaux, S. R. (2005) Undergraduate Student Perceptions Regarding Cheating: Tier 1 Versus Tier 2 AACSB Accredited Business Schools. Journal of Business Ethics, $62,407-418$.

Robinson, V. \& Kuin, L. (1999) The explanation of practice: why Chinese students copy assignments. International Journal of Qualitative Studies in Education, 12, 130210.

Stinson, M. (2003) The Little Book of Plagiarism: What it is and How to avoid it., Leeds, Leeds Metropolitan University.

Tabachnick, B. G. \& Fidell, L. S. (2001) Using Multivariate Statistics, London, Allyn and Bacon.

Taylor, D., Glaister, K. \& Sutton, A. (2007) Student Perceptions of "What is Plagiarism?" Leeds Learning and Teaching Conference. Leeds.

Yeo, S. (2007) First-year university science and engineering students' understanding of plagiarism. Higher Education Research \& Development, 26, 199-216. 
Table 1: Rotated factor loadings

\begin{tabular}{|c|c|c|c|c|}
\hline & 1 & 2 & 3 & 4 \\
\hline Let someone I trust have my completed assignment & $.805^{*}$ & .197 & .122 & -.062 \\
\hline Let someone I trust have my notes to help them & $.805^{*}$ & .221 & .013 & -.012 \\
\hline Exchange completed assignment electronically & $.582 *$ & .476 & .102 & -.040 \\
\hline Part of informal study group & .029 & $.780^{*}$ & .007 & .145 \\
\hline Exchange electronic text with study group & .165 & $.706^{*}$ & .132 & -.012 \\
\hline Put notes on social networking sites & .170 & $.594 *$ & .071 & -.212 \\
\hline Research on web with other students & .251 & $.576^{*}$ & -.058 & .192 \\
\hline Cut and paste into notes & .073 & .084 & $.693 *$ & .360 \\
\hline Work directly on screen from electronic sources & .074 & -.075 & $.602 *$ & -.080 \\
\hline Notes handwritten, only final is typed & .156 & -.041 & $-.588 *$ & .277 \\
\hline Cut and paste into final version & .235 & .194 & $.582 *$ & -.005 \\
\hline No notes, work directly on final version & .305 & -.062 & .163 & $-.678 *$ \\
\hline Ask other students to proof read my work & .487 & .015 & .104 & $.597^{*}$ \\
\hline
\end{tabular}

Note: * indicates highest factor loading 
Table 2: Descriptive statistics for factors

\begin{tabular}{|c|c|c|c|c|c|}
\hline & & & Institution A & Institution A & Institution $\mathrm{B}$ \\
\hline & & Total & Psychology & Business & Business \\
\hline \multirow[t]{2}{*}{ Trust } & Mean & 1.51 & 1.26 & 1.77 & 1.51 \\
\hline & SD & .59 & .37 & .68 & .58 \\
\hline \multirow[t]{2}{*}{ Cooperation } & Mean & 1.57 & 1.52 & 1.74 & 1.54 \\
\hline & SD & .53 & .50 & .58 & .51 \\
\hline \multirow[t]{2}{*}{ Use IT } & Mean & 2.19 & 2.08 & 2.44 & 2.15 \\
\hline & SD & .61 & .59 & .63 & .59 \\
\hline \multirow[t]{2}{*}{ Conscientious } & Mean & 3.12 & 3.07 & 3.13 & 3.13 \\
\hline & SD & .76 & .78 & .85 & .73 \\
\hline
\end{tabular}

